\title{
Sense and sensitivity in bioprocessing - detecting cellular metabolites with biosensors
}

Department of Life Sciences and Centre for Synthetic Biology and Innovation, Imperial College London, London SW7 2AZ, United Kingdom

\section{l.dekker@imperial.ac.uk}

k.polizzi@imperial.ac.uk

*Corresponding author: k.polizzi@imperial.ac.uk, T: (44) 2075942851

\begin{abstract}
Biosensors use biological elements to detect or quantify an analyte of interest. In bioprocessing, biosensors are employed to monitor key metabolites. There are two main types: fully biological systems or biological recognition coupled with physical/chemical detection. New developments in chemical biosensors include multiplexed detection using microfluidics. Synthetic biology can be used to engineer new biological biosensors with improved characteristics. Although there have been few biosensors developed for bioprocessing thus far, emerging trends can be applied in the future. A range of new platform technologies will enable rapid engineering of new biosensors based on transcriptional activation, riboswitches, and Förster Resonance Energy Transfer. However, translation to industry remains a challenge and more research into the robustness biosensors at scale is needed.
\end{abstract}

\section{Introduction}

Biosensors are a partially biological entity that can be used to monitor a parameter of interest or target molecule [1] using biological recognition elements such as proteins or nucleic acids.

Biosensors come in different forms; some are fully biological, such as whole cells (biological biosensor), and others utilise an in vitro enzymatic detection unit where information is processed by a physical/chemical component (chemical or electrochemical biosensor). Biological biosensors are genetically engineered cells or cell extracts that express a genetic mechanism for sensing the compound of interest linked to an output, often a detectable reporter protein. They tend to make use of natural sensing and signalling pathways in cells where the sensor mechanism is transcriptional, translational or post-translational. A chemical biosensor produces an electrical or optical signal proportional to the concentration of analyte usually via an enzymatic catalysis step. Numerous biosensors have been constructed using synthetic biology (reviewed in [2-6]).

Bioprocessing engineering entails translating biological science into manufacturing processes, which involves many challenges in scale up and optimisation. Monitoring the manufacturing process is vital to ensure product quality and reproducibility $[7,8]$. It requires both a systematic investigation of the best operating parameters during process development and analysis of 
cultures during production. Parameters such as the concentration of nutrients (e.g. glucose) and metabolites (e.g. lactate), reaction parameters such as $\mathrm{pH}$, pressure, temperature, oxygen and $\mathrm{CO}_{2}$ tension, and product parameters like cell density or the concentration of enzymes are typically monitored to help control the culture trajectory and ensure product quality (Figure 1, top left). Analytics are often employed during process development to better understand the relationship between external bioprocess conditions and cellular physiology. The latter is becoming increasingly important in the era of 'quality-by-design' initiatives proposed by regulators in the pharmaceutical and biopharmaceutical industries [8].

Synthetic biology has a lot to offer in the development of new biosensors for bioprocessing as it enables the rapid engineering of new biological biosensors with enhanced sensitivity, specificity, and robustness. However, the majority of biosensors currently used in the bioprocessing industries are chemical and this is also still an active area of research. In this review we discuss current trends in the development of biosensors, both biological and chemical, that can be applied to bioprocessing and critically discuss the improvements necessary to facilitate their adoption by industry. We will also review emerging trends in the development of biosensors using synthetic biology, particularly methodologies that can be exploited for developing new biosensors for small molecule metabolites. We omit biosensors for detecting cellular stress, as these have been recently reviewed elsewhere $[9,10]$.

\section{Current state-of-the-art practice in industry is to use primarily electrochemical sensors} Currently, in-bioreactor probes are used to monitor temperature, $\mathrm{pH}$ and dissolved oxygen (DO) concentration. $\mathrm{pH}$ and $\mathrm{DO}$ are generally measured using electrochemical probes or optical sensors and some contain integrated thermocouples to measure temperature [11]. For measuring key nutrients, waste metabolites, and some gases, the majority of biomanufacturers use automated amperometric biosensor devices [12], such as the BioProfile ${ }^{\circledR}$ Automated Chemistry Analyzers (Figure 1, bottom left), due to their ease of use, low cost per sample, and the ability to run a range of tests on a single sample. BioProfile ${ }^{\circledR}$ Analyzers use a combination of measurement technologies, including amperometric, potentiometric, and photometric sensors, to measure between 2 and 16 metabolites in a single sample depending on the model. In early models, high sample volumes were required $(\sim 1 \mathrm{~mL})$, but the recently released BioProfile ${ }^{\circledR}$ FLEX2 Analyzer operates with a reduced sample volume of $265 \mu \mathrm{L}$, a $75 \%$ reduction in the required volume. Currently, the main limitation with BioProfile ${ }^{\circledR}$ Analyzers is that the linear range of measurement often does not span what is needed - the lower limit of detection of some metabolites (e.g. lactate) is in the $\mathrm{mM}$ range making it impossible to monitor these across the duration of the culture. In some cases, the upper limit of detection is such that the samples require dilution, leading to extra sample handling, decreasing throughput. Despite this, the BioProfile ${ }^{\circledR}$ FLEX2 Analyzer can measure 16 metabolites in an analysis time of 4.5 minutes.

Although there has been a lot of research activity in biosensors (both chemical and biological) in the past decades, there has been little translation of the research into industry. One of the main 
experimentation. Therefore, future research should prioritise the performance characterization of biosensors in bioreactors at scale. Due to the speed and efficiency of BioProfile ${ }^{\circledR}$ Analyzers, there has been little progress in the field of biosensors for bioprocessing; however, synthetic biology techniques offer many exciting opportunities for the development of new biosensors that are robust under industrial conditions.

\section{Recently developed biosensors for bioprocessing}

Regardless of the host organism or the product, a conserved set of metabolites is often of interest. These include glucose as the primary carbon source, lactate as the primary waste metabolite, and the overall $\mathrm{pH}$ of the bioreactor, which is related to the production of lactate and ammonia. In many processes amino acids may also be monitored, particularly in recombinant protein production, where deficiencies in amino acids can reduce yield. This section reviews the development of new chemical and biological biosensors for these metabolites (Figure 1, top right).

Glucose biosensors are typically electrochemical and exploit the glucose oxidase (GOx) enzyme, which catalyses the oxidation of glucose into gluconolactone and hydrogen peroxide. Advancements in electrochemical glucose biosensors have focussed on improving the electron exchange between glucose oxidase and the electrode (reviewed in [13], e.g. [14-16]). An algorithm with a feeding strategy that allows glucose to be maintained in a customisable range in a dynamic fed-batch culture has been designed to account for errors in biomass and glucose estimation in industrial $\mathrm{CHO}$ cell cultures [17]. This can be used in conjunction with currently available biosensors to ensure rapid implementation during technology transfer and scale up.

Lactate biosensors are often electrochemical biosensors relying on L-lactate dehydrogenase (LDH) and L-lactate oxidase (LOx) as biological recognition elements; however, many wholecell biosensors also exist. Recently a whole-cell Escherichia coli lactate biosensor based on the transcriptional elements of the IIdPRD operon was used to monitor lactate levels in mammalian cell culture [18]. The biosensor could measure lactate levels as low as $100 \mu \mathrm{M}$ in a variety of different cell culture media, making it useful for routine monitoring of cell culture trajectories and the lactate concentration detected correlated with other existing analytical methods. Overall, the biosensor had a lower limit of detection than the BioProfile ${ }^{\circledR}$ Analyzer and was less expensive per sample analysed than commercially available lactate oxidase assay kits.

Amino acids are products commonly produced in bioprocessing and are critical precursors in the production of recombinant proteins. Some amino acids (e.g. glutamine and glutamate) are detected by the Bioprofile ${ }^{\circledR}$ Analyzers, while others are detected by analytical methods such as HPLC. Amino acids are one group of metabolites where biological biosensors are commonly researched. For example, transcription-based biosensors have been reported for arginine [19], phenylalanine [20], [21], branched chain amino acids [22], and threonine [23], most of which were used in the context of screening for overproducing strains, but could be adopted for bioprocessing. Also, a series of Förster Resonance Energy Transfer (FRET)-based biosensors for lysine were created using the lysine/arginine/ornithine binding protein flanked by two 
fluorescent proteins [24]. The sensors were cross validated with two other analytical assays and applied to monitoring the concentration of lysine in Corynebacterium glutamicum fermentations. The advantage of FRET sensors is that they are ratiometric, which increases the accuracy of quantification.

Optical biosensors are generally used for $\mathrm{pH}$ monitoring in small-scale bioreactors. They are cheap, do not require a separate reference sensor and can be easily miniaturized. Research has been undertaken to improve their long-term stability and expand the range of $\mathrm{pH}$ measured so they can be utilised in more applications [11]. Janzen et al. developed an acidic pH biosensor containing a fluorescent platinum-based reference dye (excitation at $505 \mathrm{~nm}$, emission at $600 \mathrm{~nm}$ ) that can conduct online measurements between $\mathrm{pH} 4.0$ and 7.0. The operational stability was shown by online $\mathrm{pH}$ monitoring $(4.5<\mathrm{pH}<5.8)$ of the anaerobic batch production of acetone, butanol and ethanol with Clostridium acetobutylicum [25]. For biological biosensors, synthetic promoter variants of the YGP1 promoter from Saccharomyces cerevisiae that are inducible at $\mathrm{pH} \leq 3$ [26] could be useful in developing novel $\mathrm{pH}$ biosensors. In addition, a synthetic mammalian $\mathrm{pH}$ sensor and $\mathrm{CO}_{2}$ transgene-control device was developed to monitor extracellular $\mathrm{pH}$ within the physiological range by rewiring the human proton-activated-cellsurface receptor TDAG8 to chimeric promoters. This $\mathrm{pH}$ biosensor, named $\mathrm{CO}_{2}$ ntrol, is controlled by gas-inducible gene expression and can be applied to biopharmaceutical manufacturing [27].

Multi-sensor platforms are smaller, simpler to use and more cost-effective than multiple single biosensors and are therefore a promising way to simultaneously monitor a wide range of parameters. A multi-sensor microfluidic system was developed for the parallel in situ monitoring of glucose, lactate, cell density and $\mathrm{pH}$ in bioprocessing using GOx and LOx amperometric enzyme sensors to monitor glucose and lactate, an impedance-based sensor to monitor cell density and a pH sensor based on capacitance-voltage measurements at an electrolyteinsulator-semiconductor structure. The biosensor was used to monitor cultures of $S$. cerevisiae and Lactobacillus acidophilus and cross validated by a commercial spectrophotometric assay (lactate/glucose), absorbance (cell density) and a commercial glass electrode $(\mathrm{pH})$ with good correlation. The design of the chip is flexible, thus it also has the possibility of the integration of additional sensors [28].

\section{Global trends in synthetic biology biosensors}

Taking a broader view of the small molecule biosensors reported since 2014 (regardless of the intended application area), some clear trends emerge. First is that the majority are based on activation of transcription ([19-21,23,29-37]), which may reflect the fact that the genetic parts for such biosensors are more easily identified. For example, libraries of promoters driving GFP expression are available for $E$. coli [38] and can be used in combination with flow cytometry to identify candidate biosensors in high throughput (e.g.[21]). Alternatively, transcriptomic or proteomic datasets can be used to identify transcripts or proteins whose abundance change in response to the molecule of interest, e.g. a recently reported biosensor for threonine [23]. In addition, recent work has developed methodology for co-opting bacterial regulators for use in 
other chassis, most notably yeast $[19,32,35]$, paving the way for rapidly engineering new biosensors from previously reported genetic elements.

Within the transcription-based biosensors there is also a trend towards engineering synthetic promoters rather than utilising natural ones [19,20,29,31-33,35,39]. This can be used to manipulate key properties of the biosensor. Using an exemplar of an arsenic biosensor, Merulla and van der Meer demonstrated that the background signal can be reduced by engineering an additional repressor binding site upstream or downstream of the promoter [31]. As a consequence, the linear range of detection was also extended. While placing the additional site downstream was more effective at reducing background signal, the optimal placement of the site upstream resulted in higher reporter fluorescence, suggesting it should be considered when signal detection is the paramount issue.

A second emerging trend is generic strategies to develop biosensors for different analytes with a minimum amount of re-engineering by focusing on modular architectures. Examples can be found in all types of biological biosensors. For transcription-based biosensors, Younger et al demonstrated the development of an orthogonal metabolite biosensor based on a metabolite binding domain fused to a zinc finger DNA binding domain [33] using an example of the BCR$A B L 1$ zinc finger and the maltose binding protein (MBP). Given that MBP is an archetypal periplasmic binding protein, it should be possible to adapt this strategy to the entire family, many of which bind molecules that would be interesting metabolites for sensing in bioprocessing.

For eukaryotic chassis, Feng et al presented a generic strategy for the production of small molecule biosensors where a destabilised binding domain is fused with a reporter, a fluorescent protein, enzyme, or DNA binding and activation domains to create a transcription factor, with the latter creating the best sensor characteristics [34]. In the absence of the small molecule, the protein is actively degraded by cells due to its instability. However, the presence of the molecule stabilizes the protein, leading to a detectable signal. Interestingly, mutations in different domains of the chimeric transcription factor appeared to be additive and mutations in the DNA binding domain identified in the context of one biosensor had a similar effect in another biosensor. Therefore, biosensors can be modularly composed of different DNA binding, activation, and ligand binding domains.

For biosensors based on riboswitches, the engineering challenge is how to place the riboswitch in the correct DNA context for maximum functionality. Recent work demonstrated a potentially generic, modular way to tune the properties of poorly functioning or non-functional switches without the need for reengineering [40], which could result in significantly decreased effort to create new biosensors. Although the work was conducted with a tetracycline responsive switch in the context of creating a genetic selection method, it could be applied to the development of metabolite biosensors as a number of metabolite responsive riboswitches exist in Nature (reviewed in [41]).

Finally, one of the biggest challenges of developing FRET biosensors, finding proteins with an appropriate conformational change upon binding, was recently addressed [42] via a concept 
called FREX (FRagment EXchange). In FREX, the fluorophores are located on two separate domains - the full-length protein with point mutations to abolish ligand binding and decrease stability and a fragment duplicated from the wild-type protein. In the presence of the molecule of interest, the fragment and the full length protein can interact, leading to detectable FRET. Although the fold changes with biological fluorophores were modest (1.3-1.8-fold), the affinity of the sensor is easily manipulated by using different fragments for the exchange. Once again, this represents a generic strategy that could be used to engineer new sensors for a variety of analytes.

\section{Conclusions and future perspectives}

The advantages of electrochemical biosensors include robustness, high sensitivity and excellent detection limits. They can also be used with small volumes and turbid samples in the presence of optically interfering absorbing/fluorescing compounds. Because of these advantages, electrochemical biosensors have been more readily translated into industry. However, using synthetic biology to develop novel biosensors for bioprocessing has great future potential. Biological biosensors display many advantages in terms of renewability and cost. They can also be used to directly gain access to intracellular concentrations without the need for destructive analysis (i.e. cell lysis) or inference from metabolic models. Since they are genetic, they can be designed directly into production organisms leading to enhanced capabilities. Rather than the production of a reporter protein, biological biosensors can be used to control the expression of an enzyme or metabolic factor to yield a self-regulating production strain. However, a remaining challenge is the adoption of new biosensors into industry. To ensure the success of biological biosensors in a bioprocessing environment, improvements are vital to shorten the analysis time, increase the shelf-life, and improve the scalability in industrial processes. For all types of sensors, multiplexing is increasingly necessary as more and more metabolite data is utilised in the development of bioprocesses. In this respect, microfluidic chips are promising for the development of multiplexed biosensors and can enable in situ measurements. In addition, bioinformatics and modelling can be used to mine new genetic or enzymatic detection elements and expand biosensing abilities through computer aided design of metabolic pathways (e.g. [43]). However, research should also focus on the effect of perturbations in the bioprocessing environments (e.g. low oxygen, fluctuating $\mathrm{pH}$ and temperature, inhomogeneities in bioreactors where mixing may not be complete) on biosensor function to facilitate adoption by industry.

259

\section{Acknowledgements}

This work was supported by an Engineering and Physical Sciences Research Council Frontier Engineering Award [EP/K038648/1]. The funders had no role in the design of the study. The authors would like to thank Terrence Lai and Dr Guy-Bart Stan for helpful discussions. 


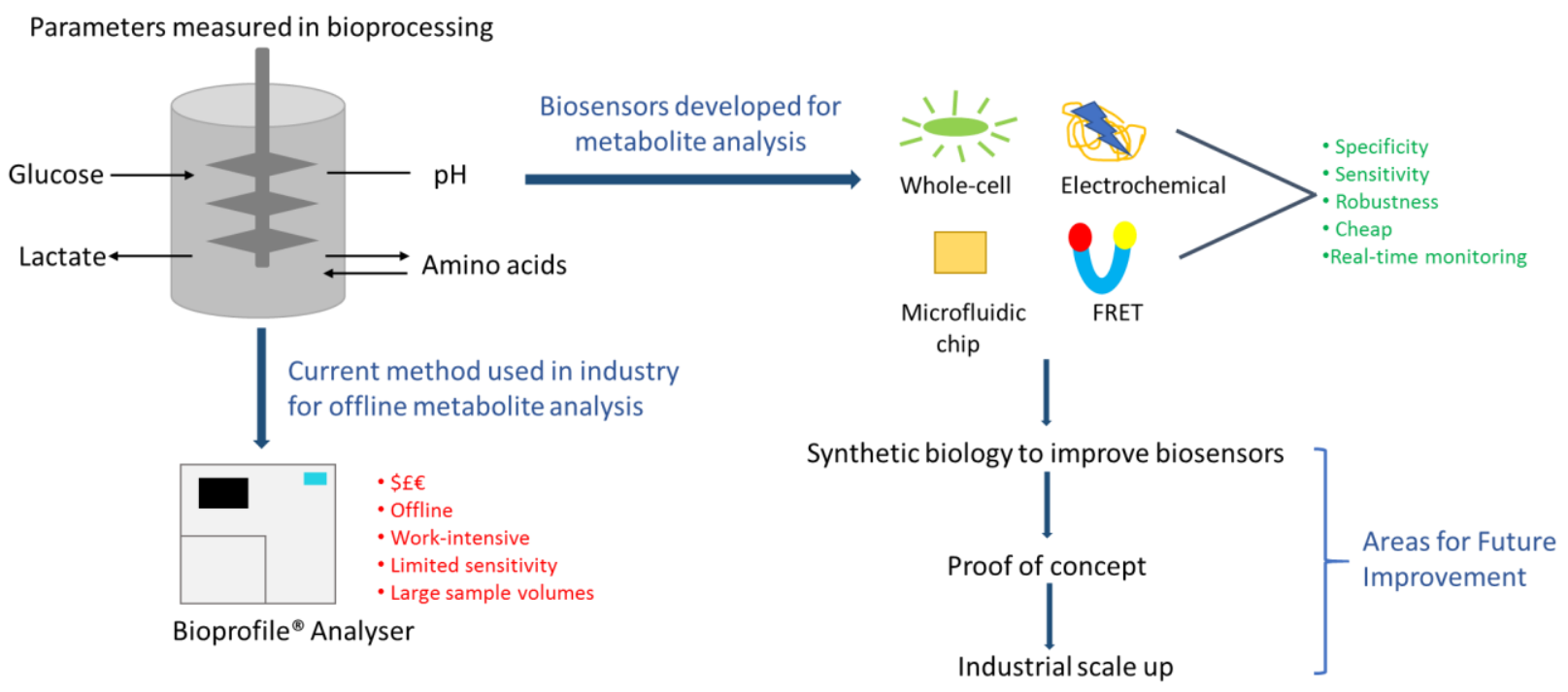

\section{Figure legend:}

Figure 1. An overview of the application of biosensors in bioprocessing. (Top left): Key metabolites that are currently monitored include glucose (primary carbon source), lactate (metabolic waste product), $\mathrm{pH}$ and key amino acids. (Bottom left): Currently industry uses instruments such as chemical analyzers (e.g. BioProfile ${ }^{\circledR}$ Analyzer), but these have disadvantages in terms of cost, sensitivity, and sample volumes. They are also off-line and rely on an operator to perform the analysis. (Top right): Development of new biosensors is a very active area of research and examples include new whole cell, electrochemical, and FRET biosensors as well as microfluidic chip-based sensors. The major aims are to increase the sensitivity, specificity, and robustness, while decreasing costs. In situ or real-time monitoring would allow for a better understanding of the process and paves the way for process control. (Bottom right): Areas where future improvement is needed include the application of synthetic biology methods for engineering new biological biosensors with improved performance. What is currently impeding industrial translation is proof-of-concept of new designs under realistic conditions to enable industrial scale-up of new technologies.

\section{References}

1. Goers L, Kylilis N, Tomazou M, Wen KY, Freemont P, Polizzi KM: Engineering microbial biosensors. In Microbial synthetic biology. Edited by Harwood C, Wipat A: Elsevier; 2013:119-156. vol 40.]

2. Slomovic S, Pardee K, Collins JJ: Synthetic biology devices for in vitro and in vivo diagnostics. Proceedings of the National Academy of Sciences 2015, 112:1442914435. 
291

292

293

294

295

296

297

298

299

300

301

302

303

304

305

306

307

308

309

310

311

312

313

314

315

316

317

318

319

320

321

322

323

324

325

326

327

328

329

330

331

332

333

334

335

336

337

338

339

340

341

3. Scognamiglio V, Antonacci A, Lambreva MD, Litescu SC, Rea G: Synthetic biology and biomimetic chemistry as converging technologies fostering a new generation of smart biosensors. Biosens Bioelectron 2015, 74:1076-1086.

4. Zhang J, Jensen MK, Keasling JD: Development of biosensors and their application in metabolic engineering. Curr Opin Chem Biol 2015, 28:1-8.

- A recent and thorough review on the application of metabolite biosensors in metabolic engineering to screen or select for high producing strains and/or dynamically regulate metabolic pathways.

5. De Paepe B, Peters G, Coussement P, Maertens J, De Mey M: Tailor-made transcriptional biosensors for optimizing microbial cell factories. J Ind Microbiol Biotechnol 2016.

6. Liu D, Evans T, Zhang F: Applications and advances of metabolite biosensors for metabolic engineering. Metab Eng 2015, 31:35-43.

7. Zhao L, Fu HY, Zhou W, Hu WS: Advances in process monitoring tools for cell culture bioprocesses. Engineering in Life Sciences 2015, 15:459-468.

8. Pais DA, Carrondo MJ, Alves PM, Teixeira AP: Towards real-time monitoring of therapeutic protein quality in mammalian cell processes. Curr Opin Biotechnol 2014, 30:161-167.

9. Delvigne F, Pêcheux H, Tarayre C: Fluorescent Reporter Libraries as Useful Tools for Optimizing Microbial Cell Factories: A Review of the Current Methods and Applications. Front Bioeng Biotechnol 2015, 3:147.

10. Polizzi KM, Kontoravdi C: Genetically-encoded biosensors for monitoring cellular stress in bioprocessing. Curr Opin Biotechnol 2015, 31:50-56.

11. Demuth C, Varonier J, Jossen V, Eibl R, Eibl D: Novel probes for $\mathbf{p H}$ and dissolved oxygen measurements in cultivations from millilitre to benchtop scale. Appl Microbiol Biotechnol 2016, 100:3853-3863.

12. Dzyadevych SV, Arkhypova VN, Soldatkin AP, El'skaya AV, Martelet C, Jaffrezic-Renault N: Amperometric enzyme biosensors: Past, present and future. IRBM 2008, 29:171180.

13. Zhu C, Yang G, Li H, Du D, Lin Y: Electrochemical sensors and biosensors based on nanomaterials and nanostructures. Anal Chem 2015, 87:230-249.

14. Han L, Shao C, Liang B, Liu A: Genetically Engineered Phage-Templated MnO2 Nanowires: Synthesis and Their Application in Electrochemical Glucose Biosensor Operated at Neutral pH Condition. ACS Appl Mater Interfaces 2016, 8:13768-13776.

15. Rakhi RB, Nayuk P, Xia C, Alshareef HN: Novel amperometric glucose biosensor based on MXene nanocomposite. Sci Rep 2016, 6:36422.

16. Gao ZD, Qu Y, Li T, Shrestha NK, Song YY: Development of amperometric glucose biosensor based on Prussian Blue functionlized TiO2 nanotube arrays. Sci Rep 2014, 4:6891.

17. Konakovsky V, Clemens C, Müller MM, Bechmann J, Herwig C: A robust feeding strategy to maintain set-point glucose in mammalian fed-batch cultures when input parameters have a large error. Biotechnol Prog 2017.

18. Goers L, Ainsworth C, Goey CH, Kontoravdi C, Freemont PS, Polizzi KM: Whole-cell Escherichia coli lactate biosensor for monitoring mammalian cell cultures during biopharmaceutical production. Biotechnol Bioeng 2017.

- A whole-cell biosensor for lactate was engineered and used for absolute quantification of L-lactate in cell culture samples from three different model bioprocesses. This is one of the few examples of accurate quantification of metabolites in complex media using whole-cell biosensors.

19. Skjoedt ML, Snoek T, Kildegaard KR, Arsovska D, Eichenberger M, Goedecke TJ, Rajkumar AS, Zhang J, Kristensen M, Lehka BJ, et al.: Engineering prokaryotic 
transcriptional activators as metabolite biosensors in yeast. Nature Chemical Biology 2016, 12:951-+.

20. Liu Y, Zhuang Y, Ding D, Xu Y, Sun J, Zhang D: Biosensor-Based Evolution and Elucidation of a Biosynthetic Pathway in Escherichia coli. ACS Synth Biol 2017.

21. Mahr R, von Boeselager RF, Wiechert J, Frunzke J: Screening of an Escherichia coli promoter library for a phenylalanine biosensor. Appl Microbiol Biotechnol 2016, 100:6739-6753.

22. Mahr R, Gätgens C, Gätgens J, Polen T, Kalinowski J, Frunzke J: Biosensor-driven adaptive laboratory evolution of I-valine production in Corynebacterium glutamicum. Metab Eng 2015, 32:184-194.

23. Liu Y, Li Q, Zheng P, Zhang Z, Sun C, Cao G, Zhou W, Wang X, Zhang D, Zhang T, et al.: Developing a high-throughput screening method for threonine overproduction based on an artificial promoter. Microb Cell Fact 2015, 14:121.

24. Steffen V, Otten J, Engelmann S, Radek A, Limberg M, Koenig BW, Noack S, Wiechert W, Pohl M: A Toolbox of Genetically Encoded FRET-Based Biosensors for Rapid ILysine Analysis. Sensors (Basel) 2016, 16.

25. Janzen $\mathrm{NH}$, Schmidt M, Krause C, Weuster-Botz D: Evaluation of fluorimetric pH sensors for bioprocess monitoring at low pH. Bioprocess Biosyst Eng 2015, 38:1685-1692.

26. Rajkumar AS, Liu G, Bergenholm D, Arsovska D, Kristensen M, Nielsen J, Jensen MK, Keasling JD: Engineering of synthetic, stress-responsive yeast promoters. Nucleic Acids Res 2016, 44:e136.

27. Ausländer D, Ausländer S, Charpin-El Hamri G, Sedlmayer F, Müller M, Frey O, Hierlemann A, Stelling J, Fussenegger M: A synthetic multifunctional mammalian $\mathrm{pH}$ sensor and CO2 transgene-control device. Mol Cell 2014, 55:397-408.

28. Mross S, Zimmermann T, Winkin N, Kraft M, Vogt $\mathrm{H}$ : Integrated multi-sensor system for parallel in-situ monitoring of cell nutrients, metabolites, cell density and $\mathrm{pH}$ in biotechnological processes. Sensors and Actuators B-Chemical 2016, 236:937-946. - A microfluidic chip-based platform for the parallel sensing of glucose, lactate, cell density and $\mathrm{pH}$ is presented. The sensor elements are electrochemical or chemical in nature, but the design is flexible and could also be used with biological biosensors. This paper is an example of the type of multiplexing that can be to detect multiple metabolites in situ.

29. Zhang J, Sonnenschein N, Pihl TPB, Pedersen KR, Jensen MK, Keasling JD: Engineering an NADPH/NADP(+) Redox Biosensor in Yeast. Acs Synthetic Biology 2016, 5:15461556.

30. Hsu CY, Chen BK, Hu RH, Chen BS: Systematic Design of a Quorum Sensing-Based Biosensor for Enhanced Detection of Metal Ion in Escherichia coli. leee Transactions on Biomedical Circuits and Systems 2016, 10:593-601.

31. Merulla D, van der Meer JR: Regulatable and Modulable Background Expression Control in Prokaryotic Synthetic Circuits by Auxiliary Repressor Binding Sites. Acs Synthetic Biology 2016, 5:36-45.

-• This paper is an illustration of how sensing performance can be improved by using synthetic biology to redesign the biosensor at the DNA level. In a transcription-based biosensor, additional repressor binding sites could be used to reduce background signal, improve the dynamic range, or increase the overall fluorescence signal, depending on where they were placed.

32. Teo WS, Chang MW: Bacterial XyIRs and synthetic promoters function as genetically encoded xylose biosensors in Saccharomyces cerevisiae. Biotechnology Journal 2015, 10:315-322. 
392

393

394

395

396

397

398

399

400

401

402

403

404

405

406

407

408

409

410

411

412

413

414

415

416

417

418

419

420

421

422

423

424

425

426

427

428

429

430

431

432

433

434

33. Younger AK, Dalvie NC, Rottinghaus AG, Leonard JN: Engineering Modular Biosensors to Confer Metabolite-Responsive Regulation of Transcription. ACS Synth Biol 2017, 6:311-325.

34. Feng J, Jester BW, Tinberg CE, Mandell DJ, Antunes MS, Chari R, Morey KJ, Rios X, Medford JI, Church GM, et al.: A general strategy to construct small molecule biosensors in eukaryotes. Elife 2015, 4.

- A generic strategy for engineering transcription-based biosensors in eukaryotic cells is presented, which uses a destabilised metabolite binding protein fused either directly to a fluorescent reporter or to DNA binding and transcriptional activation domains to make a transcription factor. The presence of the ligand stabilised the complex and allows a signal to be generated. Such a modular strategy paves the way for engineering new biosensors with speed and ease.

35. Li S, Si T, Wang M, Zhao H: Development of a Synthetic Malonyl-CoA Sensor in Saccharomyces cerevisiae for Intracellular Metabolite Monitoring and Genetic Screening. ACS Synth Biol 2015, 4:1308-1315.

36. Ganesh I, Ravikumar S, Yoo IK, Hong SH: Construction of malate-sensing Escherichia coli by introduction of a novel chimeric two-component system. Bioprocess Biosyst Eng 2015, 38:797-804.

37. Mahr R, Frunzke J: Transcription factor-based biosensors in biotechnology: current state and future prospects. Appl Microbiol Biotechnol 2016, 100:79-90.

38. Zaslaver A, Bren A, Ronen M, Itzkovitz S, Kikoin I, Shavit S, Liebermeister W, Surette MG, Alon U: A comprehensive library of fluorescent transcriptional reporters for Escherichia coli. Nat Methods 2006, 3:623-628.

39. Toussaint M, Bontemps C, Besserer A, Hotel L, Gerardin P, Leblond P: Whole-cell biosensor of cellobiose and application to wood decay detection. Journal of Biotechnology 2016, 239:39-46.

40. Rugbjerg P, Gence HJ, Jensen K, Sarup-Lytzen K, Sommer MOA: Molecular Buffers Permit Sensitivity Tuning and Inversion of Riboswitch Signals. Acs Synthetic Biology 2016, 5:632-638.

- This paper presents a generic strategy for tuning the properties of a riboswitch (and creating functional switches from non-functional ones). Instead of the riboswitch directly controlling the expression of a gene, it is used to express one half of a split transcription factor with the other half expressed by a consititutive promoter, creating a 'molecular buffer'. The strategy can be used to increase the speed at which new riboswitch-based biosensors are engineered.

41. Peselis $A$, Serganov $A$ : Themes and variations in riboswitch structure and function. Biochim Biophys Acta 2014, 1839:908-918.

42. Zheng $\mathrm{H}, \mathrm{Bi} \mathrm{J}$, Krendel $\mathrm{M}$, Loh $\mathrm{SN}$ : Converting a binding protein into a biosensing conformational switch using protein fragment exchange. Biochemistry 2014, 53:5505-5514.

43. Libis V, Delépine B, Faulon JL: Expanding Biosensing Abilities through ComputerAided Design of Metabolic Pathways. ACS Synth Biol 2016, 5:1076-1085. 